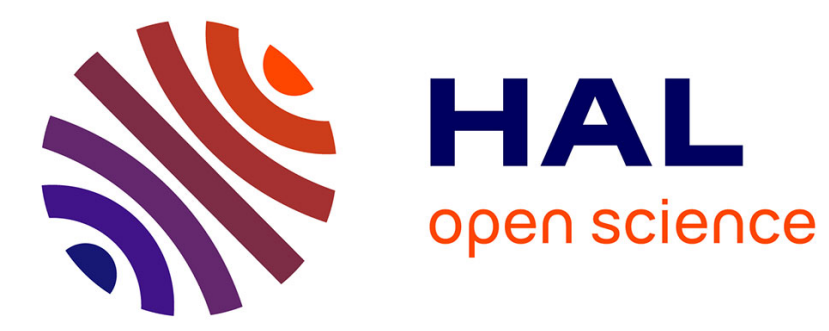

\title{
La performance des entreprises et des établissements publics
}

Melchior Salgado

\section{To cite this version:}

Melchior Salgado. La performance des entreprises et des établissements publics. Soins Cadres, 2014, 23 (92), pp.19-21. 10.1016/j.scad.2014.09.003 . halshs-02009835

\section{HAL Id: halshs-02009835 https://shs.hal.science/halshs-02009835}

Submitted on 17 May 2019

HAL is a multi-disciplinary open access archive for the deposit and dissemination of scientific research documents, whether they are published or not. The documents may come from teaching and research institutions in France or abroad, or from public or private research centers.
L'archive ouverte pluridisciplinaire HAL, est destinée au dépôt et à la diffusion de documents scientifiques de niveau recherche, publiés ou non, émanant des établissements d'enseignement et de recherche français ou étrangers, des laboratoires publics ou privés. 


\title{
L'évaluation de la performance des entreprises et des établissements publics
}

\author{
Melchior Salgado
}

\section{Résumé}

Cet article propose un travail de clarification sur l'évaluation de la performance des entreprises et des établissements publics à partir d'une revue de la littérature en sciences de gestion. Il en ressort que la performance est un concept multidimensionnel et indissociable de la création de valeur économique et sociale. De plus, ce travail met en relief l'aspect contingent de la performance liée au contexte de son évaluation : performance de quoi ? Performance pourquoi ? Performance pour qui ?

\section{Mots clés :}

Performance, valeur, stratégie, entreprises, privé, public, évaluation.

\author{
SALGADO Melchior \\ Docteur ès sciences de gestion. Habilitation à Diriger des Recherches. \\ Maître de Conférences HDR \\ Université de Lyon, Lyon 1, Labo SAF - ISFA, Associé Iseor-Magellan \\ Adresse : Bât B1, GEA, 1 rue de la Technologie, 69100 Villeurbanne Cedex \\ Mail : melchior.salgado@univ-lyon1.fr \\ Tél. : 0698859834
}

\section{Introduction}

La notion de performance est au cour de toutes les démarches d'évaluation des entreprises et des organisations. Dans les dictionnaires de langue française, la performance est définie comme un constat officiel enregistrant un résultat accompli à un instant $\mathrm{t}$, toujours en référence à un contexte, à un objectif et un résultat attendu, et ce quel que soit le domaine (Notat, 2007). Dans la pratique on constate que la performance est un mot-valise, un concept flou et multidimensionnel qui en définitive ne prend de sens que dans le contexte dans lequel il est employé.

Compte tenu de l'absence de vision partagée par les différents chercheurs sur la notion de performance ; nous proposons ici un travail de clarification sur l'évaluation de la performance à partir d'une revue de la littérature en sciences de gestion. En effet, de nombreux auteurs insistent sur l'imprécision qui entoure le concept de performance, alors que paradoxalement ce dernier constitue un des fondements majeurs des recherches effectuées en sciences de gestion, puisque leur finalité consiste à «l'amélioration des performances des organisations » (Marchesnay, 1993). De plus, les entreprises et les organisations privées ou publiques sont des acteurs et des moteurs importants de la vie économique et sociale qui explorent et exploitent en permanence des ressources pour améliorer leur performance afin d'assurer leur survie.

Voyons ci-après la diversité des regards possibles sur la notion de performance dans les entreprises. Les éléments de synthèse sur la mesure de la performance et les sources de création de valeur seront 
présentés dans un second temps. Enfin, les enjeux liés à l'évaluation de la performance dans les organisations publiques, telles que les institutions hospitalières, seront analysés dans la troisième et dernière partie.

\section{Regards croisés sur l'évaluation de la performance}

Saulquin et al. (2007), présentent trois évolutions qui permettent d'appréhender les différentes grilles de lecture de la performance.

\subsection{De la performance financière à la performance organisationnelle}

La performance a longtemps été un concept unidimensionnel, mesuré par le seul profit, en raison notamment du poids des propriétaires dans le processus de décision (Saulquin et al., 2007). Dans cette perspective, la mesure de la performance vise essentiellement la création de valeur pour les actionnaires. Il n'est donc pas étonnant que le management de l'entreprise soit centré sur cette création de valeur et la manière de la piloter. Des études récentes (Le cercle, les Echos, 2013), montrent qu'à ce jour, 200 entreprises répertoriées par la revue Fortune utilisent un indicateur basé sur la valeur créée pour les actionnaires pour évaluer la performance.

Cette logique purement financière fait aujourd'hui l'objet de fortes critiques (Dohou-Renaud, 2007 ; Bouquin, 2004), car elle n'intègre pas les différents acteurs qui participent au développement de l'entreprise (dirigeants, salariés, clients, etc.). Ainsi, pour mieux appréhender toute la complexité et la richesse du concept de performance organisationnelle, certains auteurs (Kalika, 1988 ; Morin et al., 1994) proposent une vision plus large de la notion de résultat, en proposant d'intégrer des indicateurs variés tels que la qualité du produit des produits, la mobilisation des employés, le climat de travail, la satisfaction de la clientèle, etc.

\subsection{De la performance objective à la performance en tant que construit social subjectif}

Dès lors que l'on accepte l'hypothèse de divergences des objectifs et des participants à l'organisation, le concept de performance possède autant de significations qu'il existe d'individus ou de groupes qui l'utilisent. La performance devient donc subjective, et la perception de la performance change radicalement si on se place du point de vue des dirigeants, des salariés, ou des clients.

La performance a autant de facettes qu'il existe d'observateurs à l'intérieur et à l'extérieur de l'organisation. Elle est ainsi définie par ceux qui vont utiliser l'information. Elle n'a d'importance (de valeur) que par rapport à ce que l'utilisateur de cette information va en faire. La performance reste une affaire de perception, et c'est pour cela que, dans les moments difficiles, les individus s'affrontent sur ce qu'elle est.

\subsection{De la performance "outil de mesure" à la performance "outil de management"}

Cette évolution pose la question centrale sur la finalité de la performance. En effet, la performance apparaît souvent comme un mythe mobilisateur au niveau du discours managérial (Saulquin et al. 2007); mais il n'est pas certain qu'elle soit vécue par le salarié comme un objectif individuel satisfaisant. D'un point de vue sociologique, Ehrenberg évoque le «culte de la performance » dans notre société contemporaine, et Dejours (1998) précise que la performance est un concept « rationalisateur » qui permet de tout justifier ... même des licenciements. 
L'analyse de la performance comme un simple « outil de mesure » semble donc être assez réductrice car elle s'inscrit dans une optique statique de l'entreprise, et vise prioritairement à évaluer la performance économique (Saulquin et al. 2007). Utilisée comme «outil de management », Saulquin et al. 2007 précisent que les modèles d'évaluation de la performance reposent sur une relation positive entre les pratiques managériales et des indicateurs de performance intermédiaire (comme la productivité, la qualité...), posant l'hypothèse implicite que la performance intermédiaire est gage de la performance finale.

Dans ce cadre, la performance sociale et la performance économique de l'entreprise sont deux notions indissociables. On rejoint ici le point de vue développé dans l'analyse socio-économique qui évalue la performance globale de l'entreprise en associant la performance économique à court terme (résultats immédiats), à moyen terme (création de potentiel) et la performance sociale (Savall, 1989 ; Salgado, 2012).

\section{La mesure de la performance et les sources de création de valeur.}

Voyons ci-après les logiques fondamentales qui sous-tendent l'évaluation de la performance.

\subsection{Analyse des fondamentaux des logiques d'évaluation de la performance}

Quelle que soit la grille de lecture retenue pour définir la performance, cette dernière est associé à quatre principes fondamentaux (Marion et al., 2012) :

- l'efficacité, qui traduit l'aptitude de l'entreprise à atteindre ses objectifs, en rapportant les résultats aux objectifs ;

- l'efficience, qui met en relation les résultats et les moyens, en rapportant un indicateur de résultat à indicateur de mesure des capitaux employés ;

- la cohérence, qui traduit l'harmonie des composants de base de l'organisation, en rapportant les objectifs aux moyens ;

- la pertinence, qui met en relation les objectifs ou les moyens avec les contraintes de l'environnement.

Détaillons à présent les principales sources de performance présentées dans la littérature existante.

\subsection{Les sources de performance}

Marion et al., (2012) identifient trois grands courants pour expliquer les sources de performance d'une entreprise.

Le premier concerne les travaux de recherche qui cherchent à expliquer les différences de performances constatées entre les entreprises (Rumelt, 1991 ; Roquebert et al., 1996). Dans ce courant, Marion et al. (2012) s'appuient sur les recherches de Gahan et Porter (1997) pour préciser que les caractéristiques de l'industrie et le positionnement concurrentiel des entreprises expliqueraient la moitié de la performance, mais « il n'en demeure pas moins qu'une part significative (42\%) de cette dernière reste inexpliquée ».

Le second courant concerne la théorie des ressources qui met en relief l'importance des ressources de l'entreprise, et de leur capacité pour tirer au mieux parti de ces ressources, et aussi à les régénérer (courant sur l'innovation d'exploitation et d'exploration). Dans cette optique, la performance est 
directement dépendante de l'aptitude de l'entreprise à mobiliser des ressources pour transformer à son avantage les conditions de son environnement (Barney, 1991).

En complément à la théorie des ressources, le dernier courant focalise son attention sur l'étude du rôle joué par les capacités organisationnelles. Dans cette perspective, "l'évaluation de la performance repose sur l'appréciation de la qualité des ressources et celles de leurs modes de coordination» (Marion et al., 2012).

La «formule » proposée par Calori et Atamer (2003) permet de résumer les trois principales sources de performances présentées ci-dessus : «E $=\boldsymbol{P S} \boldsymbol{x} \boldsymbol{R} \boldsymbol{x} \boldsymbol{M o e r} »$. Ici, l'efficacité (E) est le produit de la position stratégique (PS), des Ressources que peut mobiliser l'entreprise (R) et de la qualité de leur mise en ouvre (MoeR). Dans cette perspective, les auteurs signalent que la performance sera d'autant plus forte et solide dans le temps que l'entreprise saura renforcer les trois termes (PS, R, et Moer) ; mais aussi que l'un des termes peut compenser la faiblesse dans un autre champ de performance.

Voyons à présent les enjeux liés à la valorisation de la performance dans le contexte des organisations publiques.

\section{Les enjeux liés à la valorisation de la performance des organisations publiques}

\subsection{Analyse des spécificités des établissements publics}

Un établissement public est une personne morale de droit public disposant d'une autonomie administrative et financière afin de remplir une mission d'intérêt général, précisément définie, sous le contrôle de la collectivité publique dont il dépend (État, région, département ou commune). Les établissements publics sont soumis à trois principes clés:

- l'autonomie (leur organisation est très variable et ils disposent d'un budget propre lié à des subventions, des redevances des usagers, des emprunts, etc.),

- le rattachement à un niveau de l'administration (on distingue des établissements publics nationaux, rattachés à l'État, et des établissements publics locaux rattachés à une commune, un département ou une région).

- la spécialité : les domaines d'intervention des établissements publics sont variés, mais la plupart remplissent une mission de nature économique et/ou sociale. Il peut s'agir du domaine de la santé, de l'enseignement (ex : universités, lycées), de la culture, de l'économie (Caisse des dépôts et consignations, SNCF), etc.

Dans le contexte actuel de réduction des déficits, l'évaluation de la performance des établissements publics prend tout son sens. Compte tenu du fait que les finalités des entreprises publiques sont différentes de celle du secteur marchand, la création de valeur ne peut être entendue comme une recherche de profit financier (Pestieau et Gathon, 1996). En intégrant différents critères tels que la satisfaction des citoyens et celle des employés de la fonction publique, la notion de performance devient comme nous l'avons évoqué dans le première partie «multidimensionnelle ». Ainsi, à l'heure où la loi organique relative aux lois de finances (LOLF) généralise le raisonnement en termes d'indicateurs de performance, l'économie sociale doit aller vite dans la mise au point opérationnelle de nouveaux instruments de mesure de sa performance économique et sociale.

Voyons à titre d'illustration, quelques enjeux liés à l'évaluation de la performance dans le cadre de l'institution hospitalière. 


\subsection{L'évaluation de la performance dans l'institution hospitalière publique}

L'évaluation de la performance des hôpitaux s'installe progressivement dans les différentes structures de santé et devient d'une importance cruciale pour les acteurs qui travaillent dans ce domaine. Signalons à titre d'exemple, les discussions souvent enflammées sur le plan politique entre les différents ministres de la santé qui se succèdent :

- en 2009, Roselyne Bachelot préconisait de gérer l'hôpital comme une entreprise privée afin de ramener les établissements publics à l'équilibre budgétaire. Sa loi prévoyait notamment la création d'agences régionales de santé qui coordonnent les hôpitaux, la médecine de ville et le secteur médico-social.

- Et quatre années plus tard, Marisol Touraine (BFM, 4 mars 2013), Ministre de la Santé précise que l'hôpital n'est pas une entreprise : "A ceux qui veulent considérer l'hôpital public comme une entreprise, je veux dire qu'ils se trompent", et que l'enjeu est surtout de "tourner la page de la loi HPST (hôpital patients santé territoire), dite loi Bachelot, qui a été mal acceptée dans les milieux hospitaliers car elle préconisait pour l'hôpital public des méthodes de gestion plus proches de celles de l'entreprise".

Au delà des mots et du discours politicien, les questions centrales soulevées par ce débat portent bien sur l'évaluation de la performance, et sur le transfert d'indicateurs de mesure issus de l'entreprise privée vers le public. Idjadi (2013) constate qu' «une ère nouvelle est née dans les structures hospitalières : celle des gestionnaires »; et Barreau (2014) précise que «jusqu'à présent, les seules théories managériales déclinées à l'hôpital relèvent de théories généralistes le plus souvent tirées des sciences humaines ... qui manquent cruellement de pertinence pour être dupliquées en l'état ». Dans le contexte hospitalier, nous pensons que la prise en compte du concept d'utilité sociale peut utilement contribuer à concilier la performance des soins (du patient / client) et la performance économique de la structure hospitalière.

\section{Conclusion}

La performance globale se définit par des indicateurs multi-critères et multi-acteurs et non pas par une mesure en quelque sorte unique. Quelque soit l'acception retenue, l'évaluation de la performance est associée aux principes fondamentaux d'efficacité, d'efficience, de cohérence et de pertinence. Et, les travaux de recherche permettent d'identifier trois sources de performance: le positionnement stratégique, les ressources, et la capacité à mettre en œuvre les ressources.

Par rapport à l'évaluation de la performance des établissements publics tels que les institutions hospitalières, nous constatons qu'une culture de l'évaluation s'installe progressivement, et pensons que cette dernière doit simultanément intégrer trois dimensions pour prendre en compte les spécificités de ces structures : le social, le sociétal et l'économique.

\section{Bibliographie}

Barney J., «Firms Resources and sustained competitive advantage », Journal of Management, 1991 ; 17.

Barreau P., "Qu'en est-il de la recherche en sciences de gestion de l'hôpital ?", Soins Cadres, février $2014 ; 89$. 
Bouquin H., Le contrôle de gestion, Presses Universitaires de France, Collection Gestion, $6^{\text {ème }}$ édition, Paris, 2004.

Calori R. et Atamer T., Diagnostic et décisions stratégiques, Dunod, 2003.

Dejours, C., Souffrance en France, Editions du Seuil, Paris, 1998.

Dohou A., Berland N., "Mesure de la performance globale des entreprises", HALSHS, 2007.

Idjadi D., «Les paradoxes des réformes hospitalières sur la prise en charge globale du patient », Soins Cadres, $\mathrm{n}^{\circ} 86$, mai 2013.

Kalika, M., Structures d'entreprises : réalités déterminants, performances, Economica, 1988.

Le cercle Les Echos, http://lecercle.lesechos.fr/print/61804, 11 février 2013.

Marchesnay M., Management stratégique, Eyrolles, 1993.

Marion A., Asquin A., Everaere C., Vinot D., Wissler M., Diagnostic de la performance d'entreprise, Concepts et Méthodes, Dunod 2012.

Morin, E.M., Savoie, A. et Beaudin, G., L'efficacité de l'organisation. Théories, représentations et mesures, Montréal, Gaëtan Morin, 1994.

Notat N., "Une question centrale", Acteurs de l'Économie, dossier spécial performance, octobre 2007, p. 72.

Pestieau P., Gathon H.J., La performance des entreprises publiques. Une question de propriété ou de concurrence? Revue économique, 1996 ; vol. 47, nº6.

Porter M., Choix stratégiques et concurrence, Economica, 1982.

Roquebert J.A., Philipp R.L., Westfall P.A., "Market Versus Management: what drives profitability?", Strategic Management Journal, 1996 ; vol. 17, nº.

Rumelt R.P., « How much does industry matter », Strategic Management Journal, 1991 ; vol.12.

Saulquin J., Schier G., « Responsabilité sociale des entreprises et performance » Complémentarité ou substituabilité ? La Revue des Sciences de Gestion, 2007/1 n²23.

Salgado M., La fertilisation croisée des actions de production et de transmission de connaissances en management stratégique, document d'Habilitation à Diriger des Recherches en Sciences de Gestion, Université Jean-Moulin Lyon, 18 juin 2012.

Savall H., Zardet V., Maitriser les coûts et les performances câchés, Economica, 1989. 